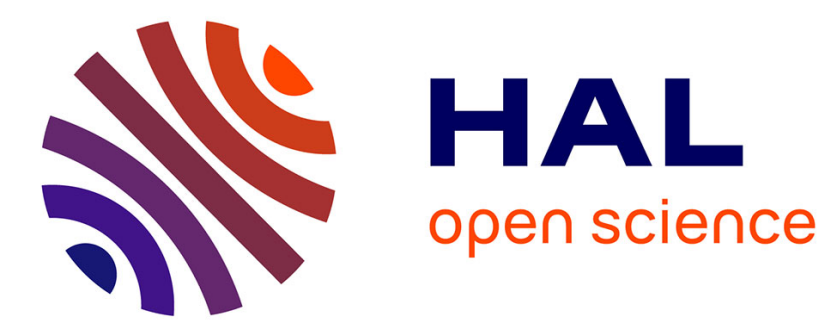

\title{
Web site search engine optimization: a case study of Fragfornet
}

\author{
A. Gandour, A. Regolini
}

\section{To cite this version:}

A. Gandour, A. Regolini. Web site search engine optimization: a case study of Fragfornet. Library Hi Tech News, 2011, 28 (6), pp.6-13. 10.1108/07419051111173874 . hal-02595571

\section{HAL Id: hal-02595571 \\ https://hal.inrae.fr/hal-02595571}

Submitted on 15 May 2020

HAL is a multi-disciplinary open access archive for the deposit and dissemination of scientific research documents, whether they are published or not. The documents may come from teaching and research institutions in France or abroad, or from public or private research centers.
L'archive ouverte pluridisciplinaire HAL, est destinée au dépôt et à la diffusion de documents scientifiques de niveau recherche, publiés ou non, émanant des établissements d'enseignement et de recherche français ou étrangers, des laboratoires publics ou privés.

$$
\text { Copyright }
$$




\section{Website Search Engine Optimization: A Case Study of Fragfornet}

4

5

\author{
Library Hi Tech News, Volume 28, Issue 6
}

Aurélie Gandour, Cemagref, Saint-Martin-d'Hères

Amanda Regolini, Cemagref, Saint-Martin-d'Hères, amanda.regolini@cemagref.fr

Keywords fummary 


\title{
Executive Summary
}

Search Engine Optimization (SEO) is a set of techniques used by websites in order to be better indexed by search engines. Here, we chose to focus upon "white hat", "in page" SEO: techniques to improve one's site content, hereby making it more attractive to human visitors as well as search engines, by making changes within the site's pages while focusing on chosen themes and keywords. The final goal is for the site to be better ranked by one or several targeted search engines and therefore appearing higher in their results lists for specified requests.

This paper describes the steps one must take to reach such a goal, while focusing on the example of the website "Fragfornet". Fragfornet is a network bringing together researchers and information professionals in order to better investigate and communicate about the issues of forest fragmentation in South America and around the world. Its website was launched at the end of 2007 and has been generated through a "website factory", allowing the creation of dynamic websites on demand, using the content management system Zope Plone, for the employees of a French public institute. Therefore, the advices provided here can be extrapolated to other websites coming from this "factory", to any website using the Zope Plone system and, even wider, to any dynamic website wanting to begin a search engine optimization.

The first effects of this SEO campaign were visible barely a week after the end of the site's optimization, achieving better rankings on pertinent keywords and bringing in more visitors. Those improvements have been successfully maintained throughout several months. The monitoring of some parameters (Google positioning of the site for selected keywords, keywords used by visitors and site's traffic) allowed us to make some observations of how to improve the site's optimization further: it is essential for such an optimization to be part of an uninterrupted, long-term work in order to be lastingly effective.

\section{Keywords}

\author{
Fragfornet \\ Search engine optimization \\ SEO \\ Google \\ Dynamic website \\ Content management system \\ CMS \\ Zope Plone
}




\section{Introduction}

Fragfornet's network intends to become a platform for researchers, stakeholders, policy makers and the public to exchange ideas, knowledge, data, experiences and results on the loss of biodiversity in the remaining native forests of South America. This network is the result of an initial workshop organized in Bariloche, Argentina: "Understanding Biodiversity Loss: A Workshop on Forest Fragmentation in South America" (26 - 30 June 2006) (Fragfornet, 2006).

In order to allow members of the network to share and spread information, a website was launched at the end of 2007 (http://fragfornet.grenoble.cemagref.fr/). Hosted by Cemagref (a French agricultural and environmental research institute), it uses the tool put at its employees' disposal, the Content Management System (CMS) Plone, based on a Zope application server.

A CMS allows, through easy-to-use interfaces, to follow the whole life-cycle of the site's web pages: their creation, their management, their publishing, etc. One of Plone's main advantages is its ability to manage a multilingual website: Fragfornet's pages are being systematically translated in English, French and Spanish. Moreover, it is a well established software throughout South America, around which a large user community has been created.

In three years of existence, Fragfornet went from 40 to 150 members. Membership enables the access to additional information, to a personal space within the website and the inscription to the network's mailing-list.

Fragfornet is a very active network. Its members organize or take part into many congresses and workshops for scientists (i.e.: Campos de Jordao, Brazil, 2009) as well as librarians (most recently: IAALD, Montpellier, France, 2010). The members of Fragfornet also published a book in 2007 (Pacha, 2007) and contributed to a book chapter published by Springer in 2009.

After a few years of existence, members of Fragfornet asked themselves what measures they could take to further improve their network's visibility, to promote its actions and publications. Though the network had an online presence, its website had a limited audience. Therefore, it was decided to try and improve Fragfornet's website visibility and an optimization of the website for its indexing by search engines was launched in 2010. This complex work allowed us to obtain quick enough results, barely a few weeks after the set up of the gathered recommendations.

Fragfornet web pages have been generated through a "website factory" allowing the creation of dynamic websites on demand for the employees of Cemagref. This paper's goal is to explain the steps to take to optimize for search engines any website website using Zope Plone; even more broadly, the general recommendations described below can be used by any website at all to gain more visibility on search engines.

After a literature review about search engine optimization, we will describe the methods we used to optimize the website before exposing the results we quickly obtained. 


\section{Definitions: Search Engines and Search Engine Optimization}

The Internet has become a wide pool of information where users dive to try and find relevant pages while webmasters fight for the visibility of their sites. In this two-sided universe, search engines are a third party, acting as intermediaries, connecting (hopefully) users to the web pages containing the information they search for.

It is useful to get a broad understanding of the functioning of a search engine (Google, 2010a; GoogleGuide.com, 2007). Initially, the engine sends robots called crawlers or spiders gather web pages and index them in huge databases. When a user click the "search" button, his query as well as the pages of the database are processed through an algorithm in order to match the most relevant pages to the user's search. Those pages, sorted by relevance, are then published as the "organic" or "natural" results of the search, usually on the left side of the page (White, 2009).

Free and easy to use, search engines tend to project an image of objectivity (White, 2009). After all, they do not promote their own content, rather pointing toward external pages, without expressing any opinion of themselves. But, behind the scene, they are not as neutral as they might seem: their popularity makes them attractive to advertisers and they are even becoming the leading advertising medium through their sponsored lists of results (usually displayed on the right side of the page (White, 2009)). By 2011, they are predicted to generate annual revenue over $\$ 45$ billion worldwide (Xu, 2009).

Having become an advertisement battlefield, literature on the use and functioning of search engines has understandably focused on the business point of view: search engine marketing management (i.e.: Heinze, 2010), search advertising (i.e.: Ghose \& Yang, 2009), auction mechanism for paid placement (i.e.: Feng, Bhargava, \& Pennock, 2007), interplay between organic and sponsored lists of results (i.e.: Xu, 2009), etc. Thus, the primary function of search engines, displaying relevant links for a user's search through its "organic" list of results, as well as the possibilities for a better indexing by the search engines of scientific, non business websites, have been overlooked and thoroughly neglected.

Search Engine Optimization (SEO) gathers the techniques leading to a better indexing of one's site by one or several targeted search engines. It seems to be mostly viewed as a subset of activities embedded within Search Engine Marketing Management (Heinze, 2010). In the best cases, papers focus on the negative (or not so negative) effects (Berman \& Katona, 2010) and search engines' strategies to deflect (Xing, 2006) what is called "black hat" SEO: different techniques that affect the search engine's quality ranking process while not affecting the quality of the web site.

Black hat SEO is the opposite of "white hat" SEO (Berman \& Katona, 2010). The latter improves one's site content, making it more relevant for visitors, and thus optimizing its indexing by search engines on chosen themes. This, of course, is the path one with honest intentions must take.

The opaqueness of the algorithms used by search engines has been thoroughly debated. Some say (White, 2009) that their utmost sophistication prevent consumers to know if the variations of results they observe for the same query ensue from "manipulative" schemes or innocent, helpful intentions. Others argue (Xu, 2009) that the mechanisms of organic ranking should stay strictly protected to prevent advertisers' manipulations. 
In spite of search engine algorithms and the global focus of literature on the SEO's business repercussions, it is possible to gather some general ideas and advice concerning the steps to take for a white hat search engine optimization of a web site. The published literature provides few articles concerning white hat SEO; therefore we had to extend our research to non-academic resources like search engines themselves, who have been known to issue recommendations to webmasters, containing useful tips towards a "white hat" optimization but also strongly warning webmasters against "black hat" optimization (i.e.: Google, 2010b). Whole companies have emerged, optimizing websites and providing counselling. Those professionals often provide usable advice through their own websites (i.e.: SEOmoz.org, 2009), or hrough specialized books (i.e.: Andrieu, 2009).

Lastly, we were most interested in consulting the few papers that report how others may have led SEO campaigns within their organizations. Those publications Some were found such as (Ho, Lu, Huang, \& Ho, 2010) who proudly report the benefits their higher rankings brought to their motel. The lack of publications presenting factual accounts of previous experiences was another motive for us to present our journey through Search Engine Optimization for a non-profit, scientific network.

Articles reporting how a business successfully marketed its brand with the help of a search engine usually advise and comment on how to use sponsor links rather than optimizing the site's ranking in organic results (McGurr, Herz, \& Stovall, 2010). But, as demonstrated by Quinton and Khan (2009), "Pay Per Click" cannot be used by organizations with very small budgets like the small and medium enterprises (SMEs) they studied. Being a non-profit, scientific association and having no budget and very few staffing to devote to SEO, Fragfornet matched this category. For this public, Quinton and Khan suggest improving the sites' ranking with a strategy including both directory submissions and press releases through specialized distribution services. Those techniques increase the number of back links for the website, improving its PageRank (a link analysis algorithm used by Google, giving more weight to a page towards which many highly ranked pages are pointing) and therefore its final ranking.

Though a high PageRank is clearly a key to a well ranked website (as demonstrated by Evans (2007)), it is possible to achieve good ranking results on specific keywords rather focusing on the page itself. This side of SEO really takes into account the now famous saying that, on the Web, "Content is King" (i.e.: Jackson, 2007). If following a few rules, a website with a rich, interesting content should have a good search engine's ranking (Andrieu, 2009). The key to a good optimization is to choose the keywords on which the site should be optimized. McMahon and Griffy-Brown \{, 2009 \#29\} conducted an online questionnaire and then selected keywords by popularity, specificity and user motivation. We preferred the simpler keyword analysis suggested by (Andrieu, 2009) using Google's tools to identify the keywords with the best potential (see below). Once the keywords have been chosen, they must be duplicated throughout the optimized pages (Zhang \& Dimitroff, 2005), and more importantly in key parts like the 'title' tag or the different headings (SEOmoz.org, 2009). The details of how to build a pertinent keyword pool and how to efficiently spread them throughout the web site are explained in the next part: "Method". 


\section{Choice of the search engines taken into account}

Although only five websites (Google, Yahoo!, Bing, Ask Jeeves and Baidu, in that order) attract $99.03 \%$ of the worldwide traffic toward search engines (StatCounter, 1999-2010, for July 2010), hundreds of alternative search engines crawl over the Web and one might ask $f$ which ones must be taken into account regarding his own site's SEO. Two types of statistics shall be observed.

\section{The first question must be: where do the current visitors come from?}

According to the tool WebStat, used to watch over Fragfornet's traffic, from august 2009 to January $2010,80.6 \%$ of the connections to the website were made through an URL directly typed within the web browser, using a bookmark or clicking a link in an email. $4.3 \%$ of the connections were made through an external page. Finally, $15 \%$ of the connections were made through a search engine. Unsurprisingly, Google is the engine most used by Fragfornet visitors. Indeed, during the second half of $2009,75.2 \%$ of the visitors having used a search engine to reach the site used Google, and $21.5 \%$ used Google Images. This leaves only $3.2 \%$ to share between all the other existing search engines.

\section{Secondly, one might ask: what search engines do the targeted audiences use?}

When looking at worldwide statistics (StatCounter, 1999-2010), Google's popularity still stands out. In July 2010, Google attracted $90.59 \%$ of the worldwide traffic towards search engines. Looking closer toward Fragfornet's targeted audiences (English, French and Spanish speaking countries), regional differences can be found (all following percentages are for July 2010). In North America, Google gathers only $83.63 \%$ of the traffic, letting $7.63 \%$ to Bing and $7.07 \%$ to Yahoo! (in the US, Google gathers $81.09 \%$ only). But in European countries (94.68\%), such as United Kingdom (92.26\%), France $(95.59 \%)$ or Spain $(97.14 \%)$, as well as in South America (97.11\% on the whole continent; $96.26 \%$ in Argentina, $98.19 \%$ in Chile), its predominance is clearer.

One of the consequences of Google's popularity is that other search engines indexing algorithms have had to recognise the preferences and indexing priorities of Google as de facto standards (Heinze, 2010). Moreover, many brands use Google's technology (AOL's search engine for example). This is why, in regard to Fragfornet's optimization, we chose to focus on its indexing by the GoogleBots: an optimization directed to them shall cover most of the targeted audience and shall also entail an improvement of the site's indexing by other search engines.

\section{Definition of a keywords' pool}

Another main step before beginning the optimization of one's site consists in the definition of a keywords' pool on which one's efforts will be focused on.

At first, a wide list of potential keywords and expressions must be established, gathering the words used to present the site's activity. It can be completed by other words found via the "related searches" from Google and the synonyms' generator of Google AdWords. During Fragfornet's optimization, this first pool of keywords was translated in the three languages used within the network (English, French and Spanish). On top of that, were added as many variations as possible (with or without accents, masculine and feminine, singular and plural).

In order to analyze those keywords' potential, two searches were made for each. Using Google Adwords, we wrote down the monthly average volume of carried out requests made upon the studied keyword. Secondly, we wrote down the volume of results found for a search on Google, on the appropriate site version (respectively, google.com, google.fr and 
google.es). From those numbers, two marks were established for each word or expression, following this scale (Table 1).

\begin{tabular}{|c|c|c|c|}
\hline \multicolumn{2}{|c|}{ Interest } & \multicolumn{2}{|c|}{ Feasibility } \\
\hline Average number of monthly requests & Mark & Number of found results & Mark \\
\hline $0-1000$ & 0 & $0-1000000$ & 20 \\
\hline $1000-10000$ & 5 & $1000000-10000000$ & 15 \\
\hline $10000-50000$ & 10 & $10000000-50000000$ & 10 \\
\hline $50000-100000$ & 15 & $50000000-100000000$ & 5 \\
\hline 100000 and above & 20 & 100000000 and above & 0 \\
\hline
\end{tabular}

Table 1: Marks awarded to keywords, translating their interest and their feasibility

Adding those two marks we obtained a global grade with a maximum of 40 . Until then, the method described ties in with Oliver Andrieu's recommendations (Andrieu, 2009). Due to Fragfornet's multilingualism, we decided to go further and add the grades obtained by each keyword in the three different languages. We thus obtained, for every variation of a word or expression, a multilingual mark, with a maximum of 120. By ordering the expressions and keywords with the multilingual grade specified above, we obtained a ranking enlightening the key expressions, both interesting, considering the potential traffic and the possibilities to be rapidly well indexed. From that list, we retained the first twenty words, whose multilingual mark was eighty-five and up, and we added a few key expressions, not as well graded but very pertinent considering the site's content. It is advised to build the indexing strategy of the website on this pool. Table 2 shows the keywords retained for Fragfornet.

\begin{tabular}{|l|l|l|}
\hline Anglais & Français & Espagnol \\
\hline Fragfornet & Fragfornet & Fragfornet \\
\hline $\begin{array}{l}\text { Forests } \\
\text { rragmentation }\end{array}$ & $\begin{array}{l}\text { Fragmentation } \\
\text { forestière }\end{array}$ & fragmentación de los bosques \\
\hline Nothofagus pumilio & Nothofagus pumilio & Nothofagus pumilio \\
\hline Deforestation & Deforestation & Deforestacion \\
\hline Landscapes & Paysages & Paisajes \\
\hline Fragmentation & Fragmentation & Fragmentacion \\
\hline Forests & Forets & Bosques \\
\hline Forested & Forestier & Forestal \\
\hline Biodiversity & Biodiversité & Biodiversidad \\
\hline Landscape & Paysage & Paisaje \\
\hline Nothofagus & Nothofagus & Nothofagus \\
\hline IALE & IALE & IALE \\
\hline Fragmentation & Fragmentation & Fragmentación \\
\hline Forests & Forêts & Bosques \\
\hline Tropical forest & Forêt tropicale & Bosque tropical \\
\hline Tropical forest & Foret tropicale & Bosque tropical \\
\hline Forested & Forestiere & Forestal \\
\hline Ecology & écologie & Ecología \\
\hline Ecology & Ecologie & Ecologia \\
\hline Cemagref & Cemagref & Cemagref \\
\hline Forested & Forestiers & Forestales \\
\hline Forested & Forestière & Forestal \\
\hline Biodiversity & Biodiversite & Biodiversidad \\
\hline
\end{tabular}

Table 2 : Pool of keywords used to optimize Fragfornet's website 
The optimization took around two weeks to put into practice (at the beginning of June 2010). We focused on the "in page" optimization, in contrast with "off page" optimization, i.e. we acted directly upon the website's pages and not on external criterions (external links leading to the site, popularity ranking of the pages, etc.). From now on, it will be possible to work on those "off page" criterions too, but the optimization of the website pages seemed like the most crucial (and most easily put in practice) option.

The "in page" optimization is what really corresponds to the "white hat" SEO. Improving the site's content, using the keywords' pool seen above, will not only benefit the web crawlers, but also the human visitors of the website .For each optimized criterion, we will first list the actual recommendations we gathered (Andrieu, 2009; SEOmoz.org, 1996-2010; etc.) and then explain what actions we effectively put into practice.

\section{$<$ title $>$ tags}

According to SEOmoz panel of experts (SEOmoz.org, 2009), keywords are the most important ones for an in-page keyword-specific ranking factor: it is crucial to choose them well and enhance them as much as possible. The <title> tag shall be written as high as possible within the HTML code. Within Fragfornet, it already appeared in the fifth line of code.

Ideally, the pages' title shall be structured as follow: [Content] - [Rubric] - [Site's name]. Before the SEO campaign, the title was worded: [Page's title] - [Site's name]. For technical reasons, we chose to transform it into: [Page's title] - [Meta description] - [Site's name]. Some of the important reasons of this choice were the ease of filling in the meta description within Plone and the near uselessness for present-day SEO of this tag.

The title shall contain 5 to 10 descriptive words. Until then, the pages' title contained an average of 2.8 descriptive words, in all of the three languages used. The addition of the meta description (containing either a short summary of the page's content or, if it was already explicit through the page's title, the title of the rubric to which the page belonged) allowed to get an average of 6 descriptive words.

\section{Visible text}

Putting aside any keyword consideration, having a substantive, unique content on the page is the most important criterion for page ranking (SEOmoz.org, 2009). Content is what contributes to the site's value. A dense, interesting and up-to-date content is the greatest asset a site could ask for, as much for its visitors as for search engines.

The vast number of Fragfornet's pages (a domain search on Google counts around 350 pages) prevented the optimization of all of the pages for visible text. First, those operations are time-costly. Second, a large number of those pages contain only links to attached files or to other internal or external pages. Thus, it seemed less important to optimize their textual content. However, it was crucial to optimize the pages already containing a lot of text: their content adds to the value of the website. They are mainly pages of the type called "Article", with wide areas of textual content. An article is a rich document containing files, images and links. It must also be taken into account that all of the site's pages do not lend themselves well to the optimization on the keyword's pool (i.e.: lists of members or partners).

The important keywords must be present at the beginning of the document, in the first paragraph. Actually, this recommendation was already put into practice by the adding of descriptive texts to the meta description tags. As it happens, those descriptions are also 
automatically displayed by Plone as an introductory paragraph of the page, at the beginning of the content's zone.

Each page must contain at least a hundred words. For example, three paragraphs were added to the homepage which initially contained only images. It brought the count to 98 words in English and up to 127 words in French.

The keywords shall be underlined to get more weight (through title tags $<\mathrm{hn}\rangle$, <strong $>$ tags or links). This recommendation too was already partly applied: the pages' titles (already containing many keywords) are automatically rewritten by Plone as editorial titles with $\langle\mathrm{h} 1\rangle$ tags at the top of the content's zone of each page. Particular attention was brought to the pool's keywords, which were underlined either with <strong $>$ and $<$ em $>$ tags or with external links pointing towards relevant web pages and PDF documents.

For a page to be optimized on a word, its keyword density must weigh between 2 and $5 \%$. This density can be calculated automatically by online software like (Keyworddensity.com, 2010), (Seochat.com, 2003-2010) or (Seobook.com, 2010). For example, after its optimization, Fragfornet's homepage now has a density of $3.21 \%$ for the keyword "forest"; the presentation page has a density of $3 \%$ for "biodiversity"; etc.

URL

378

Ideally, one shall possess their own domain name. But Fragfornet is only a sub-domain of the Cemagref's website, its URL being: http://fragfornet.grenoble.cemagref.fr. We have not been able to act upon this parameter. On the bright side, the network's name appears correctly within the site's URL, which is important for indexing.

383

384

Another important criterion is the age of the domain name: the older it is, the more reliable the site will be judged by the search engine's algorithm. Fragfornet's website exists since the end of 2007. Being a sub-domain of cemagref.fr, it shall benefit of this domain's age (which, according to Google, has been launched in January 2001).

The rest of the URL shall contain important keywords. On Fragfornet's website, the files and sub file names answer two needs: they shall be explicit but they also shall be short enough since they appear automatically in the left menu. That is why, when trying to add up some keywords to the <title> tags, those titles were not subjected to any modification. As a matter of fact, those titles had already been subjected to an optimization during the site's structure overhaul during summer 2009.

The entire URL shall avoid any accentuated character and shall be written in lower case only. Plone's URL generator already took those recommendations into account.

Lastly, the pages' titles shall match the editorial title. This recommendation is also met since Plone carries the page's title in the first part of the <title $>$ tag as well as in the file's title appearing in the URL and as an editorial title with $<\mathrm{h} 1>$ tags at the top of the content area of the page.

\section{Meta tags}

403 The meta description tag can eventually be used by Google as an abstract of the page instead of taking excerpts of the page's content (Krishnan, 2007). The tag must contain real sentences, related to the page's content, eventually naming the author and date, and have a size between 150 and 200 characters, without any CR. Those rules clashed with the diverted goal we assigned those tags for Fragfornet (bringing extra keywords to the <title> tag). Yet, the <title> tag shall not size over 100 characters. Thus, it is not possible meet both uses. The original use of the meta description tag was not useful for the site's SEO and it was decided to privilege its new use and the rules it must follow. 
Nowadays, the meta keywords tag is not taken into account by search engines. Thus, we did not fill it in.

The "lang" option allows pointing out the language in which meta tags are written. It is not automatically generated by Plone and does not seem essential since the page's language (our pages staying, as much as possible, unilingual) is already specified in the html tag.

\section{Alt and Title attributes}

420

\section{$437 \quad$ Results}

ALT is one of the IMG tag attribute. It is taken into account by Google but not by Yahoo orBing (Andrieu, 2009). In Plone, it corresponds to the "Alternative text" field in the image properties. In order to optimize those tags, they were filled within all articles containing descriptive images in order to further highlight some of the pool's keywords.

Fragfornet's website also contains a large number of photos of its members, particularly in the pages presenting its coordinators and active members. Optimizing the ALT attribute for those images, we hoped to catch more visitors doing searches on those people (it was already the case, some of the keywords used to reach Fragfornet sometimes being names of researchers or librarians.. Moreover, the ALT attributes give us the opportunity to write some keywords without accentuation as a way to catch search engines users searching badly spelled keywords. Accordingly, most of the ALT attributes of the website were also written without accents.

TITLE is another of the IMG tag attributes. It is never taken into account by any search engines, and thus it is useless for the site's SEO.

We did not have to wait long to experience the first effects of our SEO campaign. One week later, as soon as the Googlebots had crawled our site and stored a newer version of it within their databases, our site immediately went up in the results pages for requests concerning forests fragmentation. Here are some of the parameters we monitored and some of the conclusions we drew from it.

\section{Google positioning}

Since the beginning of Fragfornet's optimization procedures, the site's position for specific requests on Google was monitored. The requests used were simply the pool's keywords on the appropriate site version for each language The observed variations may be due both to the modifications made on our website and to the evolution other websites.

We first observed that for many keywords the site stayed invisible to users typing those requests, never appearing in the first one-hundred results. Those keywords were very general, unspecific words, some recovering more than one theme. For example, a search on the word "fragmentation" brings back more results about computer breakdowns than foresting issues. .

Nevertheless, Fragfornet did appear in the first one-hundred results on a few important keywords, in different languages, on Google. Those keywords are displayed in Table 3. 


\begin{tabular}{|l|l|l|}
\hline \multicolumn{1}{|c|}{ English } & \multicolumn{1}{c|}{ French } & \multicolumn{1}{c|}{ Spanish } \\
\hline FragForNet & FragForNet & FragForNet \\
\hline Forest fragmentation & Fragmentation forestière & Fragmentación de los bosques \\
\hline- & Nothofagus & Nothofagus \\
\hline Nothofagus pumilio & Nothofagus pumilio & \\
\hline IALE & IALE & IALE \\
\hline Cemagref & \multicolumn{1}{|c|}{-} & Cemagref \\
\hline
\end{tabular}

Table 3 : Keywords on which Fragfornet has been successfully optimized

Those keywords either identify the website (its name, its subject, its institution - all of which were critical points to optimize) or are very specific keywords related to the site's theme. This further stresses the need to focus on complex, specific expressions closely related to the network's activities.

We can observe that Fragfornet's position dramatically increased just after its optimization but dropped down in the following weeks. During the following months, the site's ranking varied widely, improvements often showing under the impulse of news updates. In order to be lastingly effective, optimization shall be an uninterrupted, long-term work. If the website is not updated enough, it will be labelled as unreliable by search engines' algorithms. Other websites on the same theme are likely to be optimized too, competing with each other in popularity and high ranking. SEO needs to be a continuous effort so as not lose visibility in which potential visitors go to other websites.

\section{Keywords used by our visitors}

Using specific software, it is possible to monitor, among other criteria, which keywords visitors use to reach our website through search engines. This kind of data allowed us to note that the SEO campaign for Fragfornet's website that visitors use three times more keywords from our pool than before, among the first 40 most used. In the top keywords, we find many generic words such as "biodiversity", "fragmentation", "ecology", etc. for which Fragfornet had stayed invisible for simple requests. A closer look underscores that those keywords were effectively used in more complex requests such as "Brazil biodiversity loss" or "koala landscape fragmentation", stressing again the importance of complex expressions.

Those expressions are also taken into account by our monitoring software. Their analysis reveals that the most used expression is actually a single word: "fragfornet" (and it has always been this way, the site being "naturally" optimized for its own name). Overall, the percentage of expressions used by visitors to reach our website through search engines containing at least one keyword from the keywords' pool defined above has increased gradually since the beginning of Fragfornet's optimization (see: Table 4). 


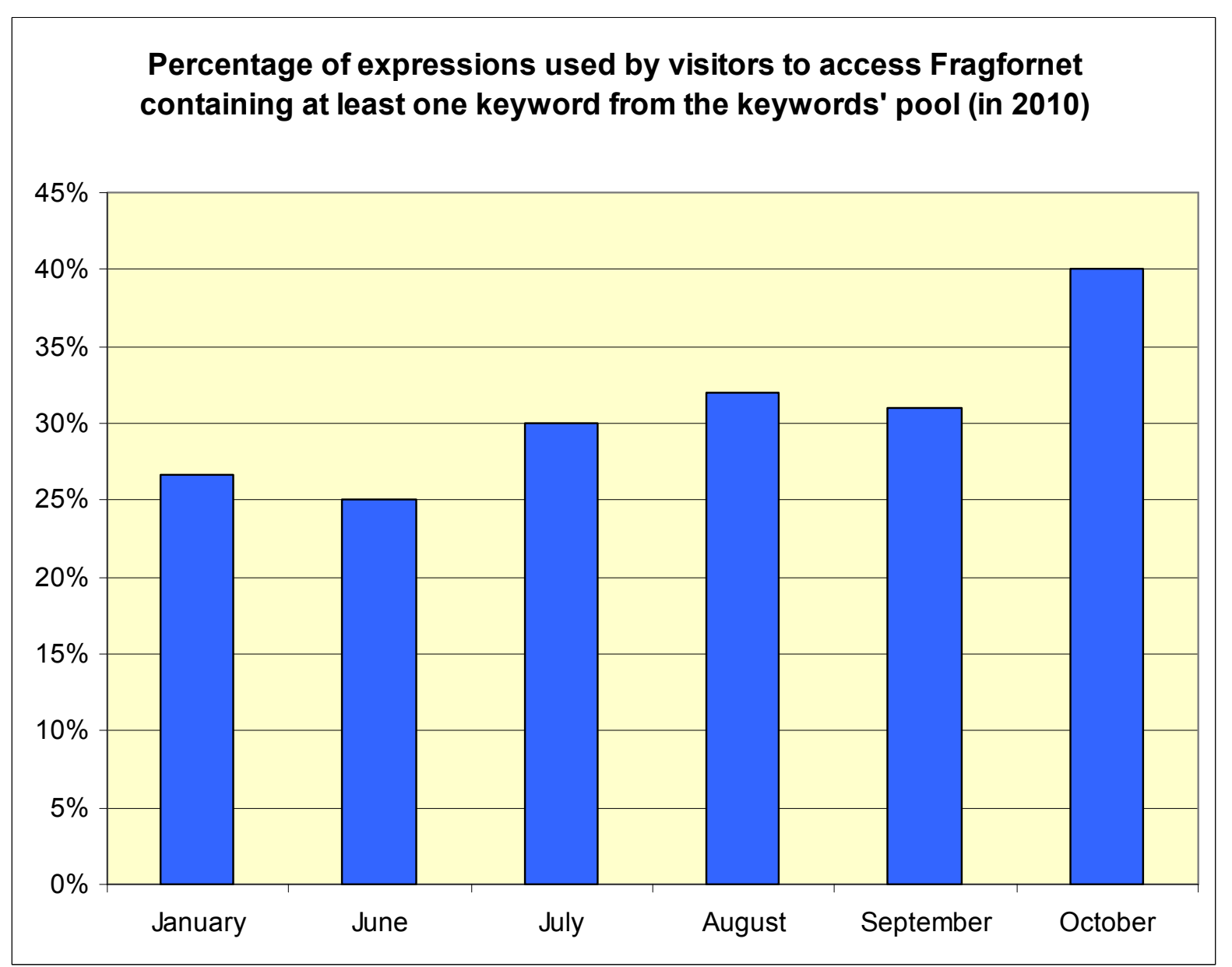

Table 4: Percentage of expressions used by visitors to access Fragfornet containing at least one keyword from the keywords' pool

This increase in keywords from our pool used by visitors to reach Fragfornet also shows in the lists of keywords issued by our software for each month. As seen in Table 5 , the site's optimization caused a raise in the percentage of keywords from the pool; it was only $3 \%$ in January and as stabilized around $6 \%$ after our campaign.

504

\begin{tabular}{|c|c|c|c|c|c|c|}
\hline $\mathbf{2 0 1 0}$ & January & June & July & August & September & October \\
\hline $\begin{array}{c}\text { Keywords from the } \\
\text { pool }\end{array}$ & $3 \%$ & $4 \%$ & $6 \%$ & $6 \%$ & $5 \%$ & $6 \%$ \\
\hline
\end{tabular}

Table 5: Percentage of keywords from the pool among the keywords used by visitors to access Fragfornet

Another important finding is the radical increase of visitors coming in after searching requests containing the names of some members of our network. The number of clicks for such name almost doubled between January and July. This increase is probably the result of the particular care we took in enhancing the names of the participating members.

\section{Site's traffic}

513 Historically (as shown in Table 6), the website's traffic varies a lot, according to the period of the year or the events to which Fragfornet's members participate. 


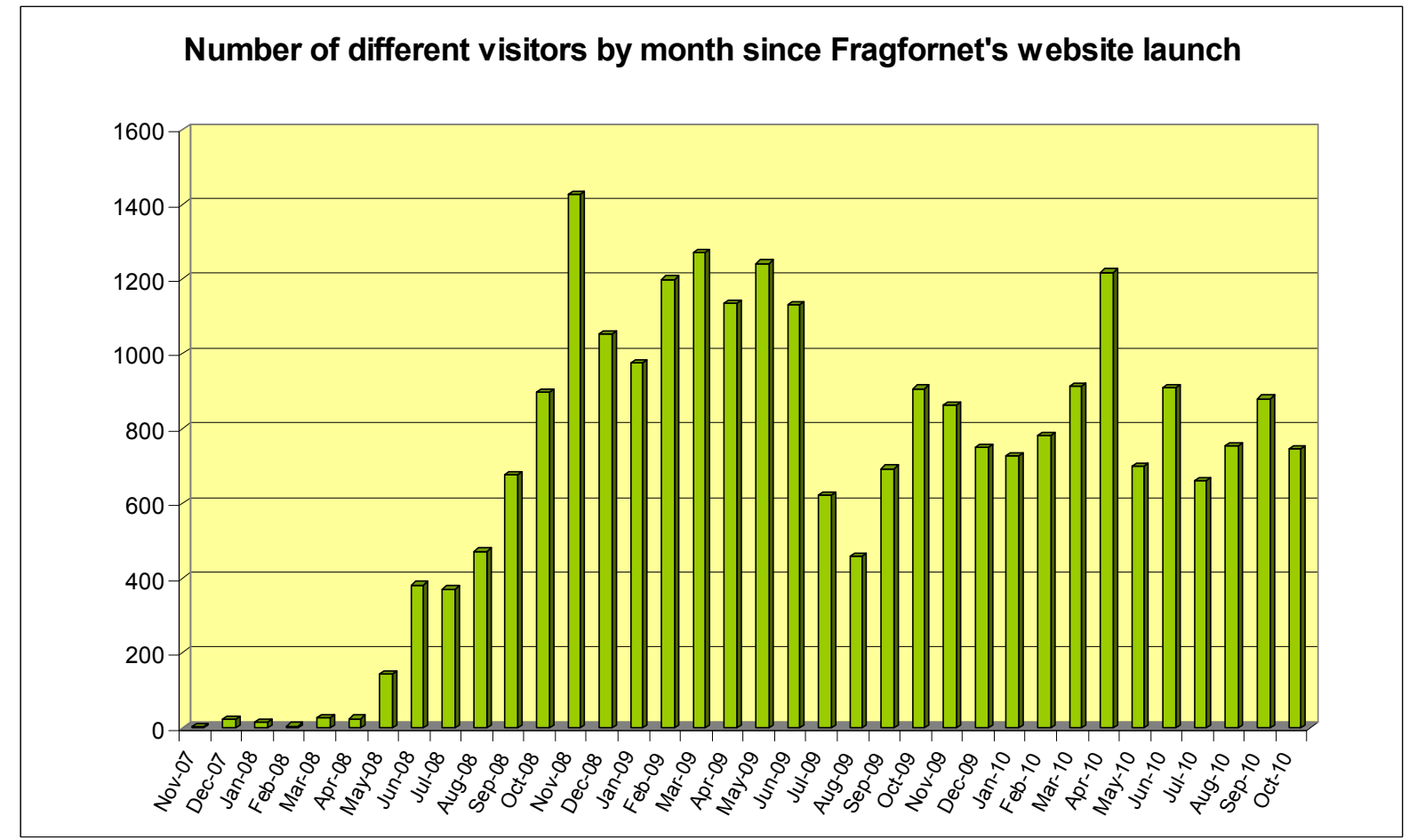

Table 6: Number of different visitors by month since Fragfornet's website launch

Nevertheless, we were able to observe an augmentation of the site's frequentation for June 2010 relative to May 2010, eventhough this tendency was reversed in 2009. This tendency was confirmed in the following months (see: Table 7): in July, August and September, 2010's traffic improved in regards of 2009. As such, the optimization of the website benefited the network with an increase in its traffic. The numbers attained are still humble but they do match the very specific scientific domain aimed by Fragfornet's network. We shall continue to monitor Fragfornet's traffic, since it is a precious witness of the site's indexing evolution. 


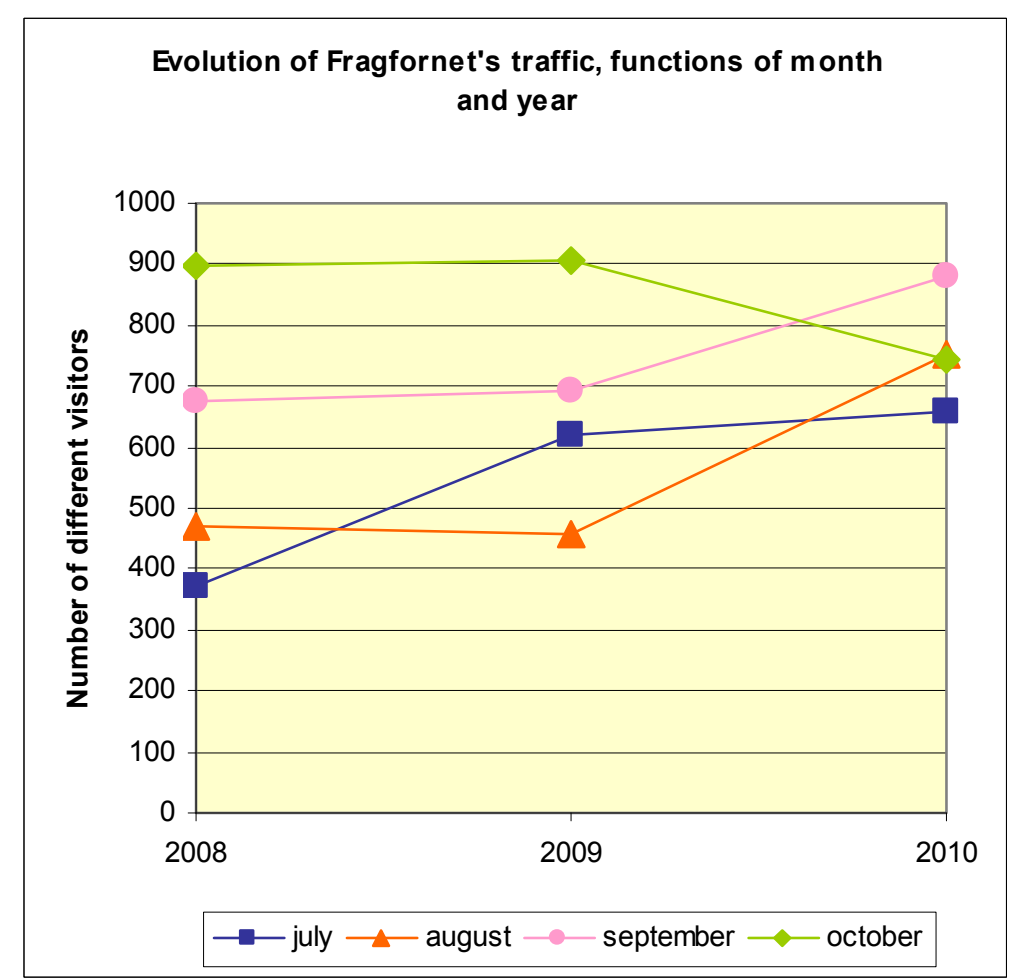

Table 7: Evolution of Fragfornet's traffic, functions of month and year

\section{Conclusion}

534

535

536

537

538

539

540

541

542

543

544

545

546

547

548

549

550

551

552

553

554

555

556

557

558

559

Ideally, SEO should be incorporated at the very beginning of a website's project planning. This article demonstrates that it is still possible to overcome a lack of visibility way after a site's launching. This work should be carried out during the whole life of the site so as to maintain a good indexing vlaue. For a site to be attractive as well for web crawlers as for human visitors, it must simply offer interesting and regularly updated content.

Because Fragfornet is not a commercial website we have to keep in mind that ood indexing is not a goal in itself. Still, some improvement of the site can still be considered for the coming months. This can involve updating the site on a regular basis, focusing on the inclusion of pertinent complex expressions and specialized keywords. The conformity towards the W3C (w3.org, 1994-2010) standards could also be improved: if Fragfornet is currently in compliance for its HTML code, it is not the case for CSS and JavaScript.

Considering "off page" criterions, it is essential to take into account the external links leading to the site. The more numerous they will be, and the more popular the pages they are coming from, the better will be the site's indexing rank. . A links' exchange campaign, though delicate to put into practice, shall allow still better indexing. We can already observe that 35 websites currently point toward Fragfornet's homepage, some of them coming from important institutions within the forest fragmentation field.

Finally, it is necessary to keep surveying the website by its visitors, keywords used, and positioning on Google (for a new keywords and expressions' pool containing more complex and specific terms). This data will allow the evaluation of the necessary efforts to maintain Fragfornet's visibility and continue its search engine optimization needs. 


\section{References}

561

562

563

564

565

566

567

568

569

570

571

572

573

574

575

576

577

578

579

580

581

582

583

584

585

586

587

588

589

590

591

592

593

594

595

596

597

598

599

600

601

602

603

604

605

606

607

608

609

610

611

612

613
Andrieu, O. (2009). Réussir son référencement Web, 2è édition. Paris: Eyrolles.

Berman, R., \& Katona, Z. (2010). The Role of Search Engine Optimization in Search

Rankings.

Evans, M. (2007). Analysing Google rankings through search engine optimization data. Internet Research, 17(1), pp. 21-37.

Feng, J., Bhargava, H., \& Pennock, D. (2007). Implementing Sponsored Search in Web Search Engines: Computational Evaluation of Alternative Mechanisms. INFORMS Journal on Computing, 19(1), pp. 137-148.

Fragfornet. (2006). Understanding Biodiversity Loss: A Workshop on Forest Fragmentation in South America. Retrieved 05/08/2010, 2010, from

http://fragfornet.grenoble.cemagref.fr/research-

activity/conferences workshops/events/introduction

Ghose, A., \& Yang, S. (2009). An Empirical Analysis of Search Engine Advertising:

Sponsored Search in Electronic Markets. Management Science, 55(10), pp. 1-18.

Google. (2010a). How Google Works. Retrieved 05/08/2010, 2010, from

http://www.google.com/howgoogleworks/

Google. (2010b, 04/06/2010 ). Webmaster Guidelines. Retrieved 05/08/2010, 2010, from

http://www.google.com/support/webmasters/bin/answer.py?answer=35769

GoogleGuide.com. (2007, 02/02/2007). How Google Works ?, from

http://www.googleguide.com/google works.html

Heinze, A. (2010). From Search Engine Optimisation to Search Engine Marketing

Management: development of a new area for information systems research. In $\mathrm{G}$. Fletcher (Ed.), UK Academy for Information Systems 15th Annual Conference 2010. Oriel College, University of Oxford.

Ho, L., Lu, M., Huang, J., \& Ho, H. (2010). The Application of SEO for Internet Marketing: An Example of the Motel Websites, Computer and Automation Engineering (ICCAE) 2010.

Singapore: IEEE.

Jackson, M. (2007, 01/05/2007). The Golden Rule of SEO: Content is King. Retrieved

05/08/2010, 2010, from http://searchenginewatch.com/3625720

Keyworddensity.com. (2010). Keyword Density Analyzer tool for search engine optimization and internet marketing experts. Retrieved 05/08/2010, 2010, from

http://www.keyworddensity.com/

Krishnan, R. (2007, 27/09/2007). Improve snippets with a meta description makeover.

Retrieved 05/08/2010, 2010, from

http://googlewebmastercentral.blogspot.com/2007/09/improve-snippets-with-meta-

description.html

McGurr, P., Herz, P., \& Stovall, S. (2010). Advertising on Google.eu: A Missed Opportunity for Colorado Ski Resort, Combi 2010. Vantaa, Finland.

McMahon, D., \& Griffy-Brown, C. (2009). Developing an effective and affordable search engine marketing strategy for nonprofits. International Journal of Internet Marketing and Advertising, 5(1-2), pp. 113-130.

Pacha, M. J. (2007). Understanding biodiversity loss: an overview of forest fragmentation in South America: International Association of Landscape Ecology, IALE.

Quinton, S., \& Khan, M. (2009). Generating web site traffic: a new model for SMEs. Direct Marketing: An International Journal, 3(2), pp. 109-123.

Seobook.com. (2010). Free Keyword Density Analyzer Tool. Retrieved 05/08/2010, 2010, from http://tools.seobook.com/general/keyword-densityl

Seochat.com. (2003-2010). Keyword Density - SEO Tools - Search Engine Optimization, Google Optimization. Retrieved 05/08/2010, 2010, from http://www.seochat.com/seotools/keyword-densityl 
614 SEOmoz.org. (1996-2010). SEOmoz. Retrieved 05/08/2010, 2010, from

615 http://www.seomoz.org

616 SEOmoz.org. (2009, 2009). Search Engine Ranking Factors 2009. Retrieved 05/08/2010,

617 2010, from http://www.seomoz.org/article/search-ranking-factors

618 StatCounter. (1999-2010). GlobalStats. Retrieved 05/08/2010, 2010, from

619 http://gs.statcounter.com/

620 w3.org. (1994-2010). World Wide Web Consortium (W3C). Retrieved 05/08/2010, 2010, from

621 http://www.w3.org

622 White, A. (2009). Search Engines: Left Side Quality versus Right Side Profits.

623 Xing, B. (2006). Internet Search Engine Result Diversity, Relevance, Quality, and the Effects

624 of Search Engine Optimization. Unpublished Doctoral Dissertation, The Rawls College of

625 Business Administration, Texas Tech University, Lubbock.

626 Xu, L. (2009). Too Organic for Organic Listing? Interplay between Organic and Sponsored

627 Listing in Search Advertising. In J. Chen (Ed.) (pp. 38).

628 Zhang, J., \& Dimitroff, A. (2005). The impact of webpage content characteristics on webpage

629 visibility in search engine results (Part I). Information Processing and Management(41), pp.

630 665-690.

631

632 